Title:

Improving the robustness of the Comparison Model Method for the identification of hydraulic transmissivities

Authors:

Alessandro COMUNIAN (alessandro.comunian@unimi.it) and

Affiliation:

Mauro Giudici (mauro.giudici@unimi.it)

Dipartimento di Scienze della Terra “A.Desio”, Università degli Studi di Milano, Italy

This is the pre-print version of the manuscript that was accepted for publication in Computers \& Geosciences, Elsevier, with the following DOI: https://doi.org/10.1016/j.cageo.2021.104705 


\section{Highlights}

Improving the robustness of the Comparison Model Method for the identification of hydraulic transmissivities

Alessandro Comunian,Mauro Giudici

- Approaches to improve the robustness of a direct inversion method are explored

- Guidelines are provided to deal with the numerical issues related to small gradients

- A tomographic approach allows to improve the robustness of the inversion procedure

- An open source implementation of the comparison model method is provided 


\title{
Improving the robustness of the Comparison Model Method for the identification of hydraulic transmissivities *
}

\author{
Alessandro Comunian ${ }^{a, *}$, Mauro Giudici ${ }^{a}$ \\ ${ }^{a}$ Università degli Studi di Milano, Dipartimento di Scienze della Terra "A.Desio", via Cicognara 7, 20129 Milano, Italy
}

\section{ARTICLE INFO}

\section{Keywords:}

Inverse Problems

Comparison Model Method

Hydraulic Transmissivity

Parameters Identification

\begin{abstract}
A B S T R ACT
The Comparison Model Method (CMM) is a relatively simple and computationally efficient direct method for the identification of the transmissivity of a confined aquifer by solution of an inverse problem. However, it suffers some of the classical drawbacks related to ill-posedness and ill-conditioning of inverse methods. Effectiveness of the CMM can be improved by some approaches. First of all, the introduction of a factor which permits to limit the negative effects of small hydraulic gradients by selection of a single parameter of the algorithm. Moreover, the CMM can be cast in a tomographic framework, i.e., by profiting of multiple sets of data, corresponding to different flow situations, produced by different boundary conditions or sources terms. Numerical tests are performed on a synthetic aquifer, by means of an open-source implementation based on the use of flopy for the solution of the forward problems. The tests show that the above mentioned approaches improve the robustness of the CMM with respect to error on the input head data.
\end{abstract}

\section{Introduction}

The identification of the spatial distribution of aquifer transmissivity is crucial to model groundwater flow and transport in aquifers. This operation is still challenging in practical applications; the solution of an inverse problem is the most rigorous approach, but is nevertheless affected by several difficulties, related to ill-posedness (non-uniqueness and instability) and ill-conditioning (Giudici, 2003; Giudici et al., 2008, 2019). In the last couple of decades, some reviews or comparison tests have been published on this topic (de Marsily et al., 2000; Carrera et al., 2005; Hill and Tiedeman, 2006; Kitanidis, 2007; Franssen et al., 2009; Zhou et al., 2014; Linde et al., 2015).

The variety of approaches proposed to tackle inverse problem can be divided in two main groups: direct approaches and indirect approaches (Neuman, 1973). Direct approaches are based on manipulations of the forward problem equation, and are often based on the assumption that the state of the system can be interpolated on the whole domain of interest and on some additional restrictions (Giudici et al., 2019). Quite often, they rely on the availability of data sets for different flow conditions. One example of these approaches is the Differential System Method (Parravicini et al., 1995; Giudici et al., 1995); this method allows to identify the transmissivity $T$ of a confined aquifer by combining two or more sets of data (piezometric heads and source terms) and at least one value of $T$ at one point of the simulation domain. Another example of direct approach is the Double Constraint Method (Brouwer et al., 2008; El-Rawy et al., 2014), where the results of two forward problem runs, one that uses pressure head constraints and one using flow rate constraints, and a prior estimate of the permeability field, are combined in an iterative fashion to identify the hydraulic permeability field of an aquifer.

On the other side, and most commonly, inverse problems are cast in an indirect approach. The indirect approach usually exploit the information available only on a limited set of locations, and an optimization method to minimize an objective function. A vast literature exists on indirect approaches (Zhou et al., 2014; Linde et al., 2015); UCODE (Poeter and Hill, 1999) and PEST (Doherty, 2015) are among the most commonly used tools.

In general, direct approaches are frugal in terms of computational resources because they only require one or few runs of the forward model, in addition to some processing of the results and the preliminary step to interpolate data on the discretization grid. On the other hand, indirect approaches require a substantial number of forward problem

\footnotetext{
${ }^{\star}$ Both AC and MG designed the research and discussed the results. AC wrote the first draft of the manuscript, developed and run the computer code. MG contributed to the design of the tests and to the revision of the manuscript. Both AC and MG equally contributed to the discussion of the results and to the final version of the manuscript.

@alessandro.comunian@unimi.it (A. Comunian); mauro.giudici@unimi.it (M. Giudici)

ORCID(s): 0000-0002-6293-404X (A. Comunian); 0000-0002-6703-5748 (M. Giudici)
} 
(model evaluation) runs. Despite the growing capabilities of parallel computing, dealing with the high number of forward problem runs remains an open research question. A possible solution are hybrid optimization methods, that blend in a smart way global and local optimization methods, as proposed for example by Vesselinov and Harp (2012). Another possibility is to find a proxy or a surrogate for the forward model, as proposed for example by Dagasan et al. (2020) with the use of Generative Adversarial Networks. Alternatively, one can also look for improving the robustness of direct approaches, as it is done in this work.

In this paper, the Comparison Model Method (CMM) is considered. The CMM is a direct inversion method first proposed by Scarascia and Ponzini (1972). Its basic properties and further improvements are described by Ponzini and Lozej (1982); Ponzini and Crosta (1988); Ponzini et al. (1989). In real cases applications, the CMM was used to identify the parameters of coastal aquifers (Giudici et al., 2012; De Filippis et al., 2016, 2017, 2019), of regional models to support groundwater-surface water interactions (Baratelli et al., 2016), of hydrogeological models of highly irrigated alluvial plains Vassena et al. (2008, 2012). Moreover, its applications are not limited to hydrology, as the method was successfully applied by Lesnic (2010) to determine the flexural rigidity of a beam and by Ponzini et al. (1989) to identify thermal conductivities. In addition, the CMM was also included in the groundwater modeling software YAGMod (Cattaneo et al., 2015). Some recent developments of the CMM include an hybrid approach that blends this direct inversion method with multiple-point statistics (Comunian and Giudici, 2018).

The CMM is a direct method of inversion based on an auxiliary model, hereinafter called Comparison Model (CM), and on the knowledge of the head field and of the source terms throughout the whole domain. The CM shares the same boundary conditions and source/sink terms with the predictive model to be developed, but the transmissivity field corresponds to a first guess, possibly informed by the results of field tests, geological or geophysical information. Basically, the CMM estimates the transmissivity field by correcting the initial guess with the ratio of the hydraulic gradients computed from the solution to the forward problem for the CM and the reference hydraulic gradients. The basic physical idea behind such an approach is that, if the initial guess is not far from the real transmissivity field, the flow rates computed with the $\mathrm{CM}$ should be a reasonable approximation of the real ones, obtained as the product of the real transmissivity times the reference hydraulic gradient.

Similarly to any other inverse method, the CMM suffers from some drawbacks. Some of the most important problems are related to the good knowledge of the head field and to the non-boundedness of derivative operators. The inverse problem is very prone to errors on heads and, in particular, even very small errors at short wavelength might induce huge errors in the estimate of the hydraulic gradient and finally of the transmissivity field.

Like other direct methods of inversion, e.g., the Differential System Method (Parravicini et al., 1995; Giudici et al., 1995; Giudici and Vassena, 2006), the head field has to be known everywhere. Since measurements are available at possibly few, sparse points, an interpolation is necessary. Often, interpolation can be a critical step: for example, if one uses kriging, the available data might not be sufficient to build up a reliable variogram model, and the errors made in the model selection will affect the interpolated head field. In order to reduce the uncertainty intrinsic in this procedure, it is good practice to use any other additional information, e.g., no flow conditions related to the outcropping of impermeable rocks. Furthermore, interpolation errors add themselves to measurement errors at the monitoring points. This might be critical, above all, in areas of low gradients, because the relative error in those areas could be particularly great, and in extreme cases the estimated hydraulic gradient could show a direction opposite to the real one.

Moreover, it should be recalled that the balance equation acts as a low pass filter for wave number components of the head field (Giudici and Vassena, 2008). In other words, the high wave number (short wavelength) components of the transmissivity field are filtered out by the balance equation, so that the head field does not carry correct information about the variability of the transmissivity field at short distances. This aspect was also investigated by Comunian and Giudici (2018), where the CMM was used in conjunction with the Direct Sampling multiple-point statistics simulation method (Mariethoz et al., 2010) in a hybrid approach to improve the reproduction of small scale details.

In order to overcome some of these difficulties, the approaches considered in this work are twofold.

First, the correction initially proposed by Ponzini and Lozej (1982) and lately improved by Vassena et al. (2012) to limit the heavy effects of low hydraulic gradients is considered. In particular, the factor $\beta$ which was introduced in the aforementioned studies is defined in a more precise and general way in this paper. This factor depends on a coefficient $\gamma$, which permits to tune the amount of cells where low hydraulic gradients should be corrected. Besides studying the implications of different choices of $\gamma$, in this work a straightforward criterion to select this parameter based on the percentage of cells where the hydraulic gradient has to be corrected is proposed.

Second, the CMM can be cast in a "tomographic" framework, by profiting of the availability of multiple data sets related to different flow conditions. If this is the case, the forcing of the natural system in such a way as to 
produce different flow directions, which is the basic idea of any tomographic approach, might be useful to reduce the dramatic effects of the data noise and to estimate a transmissivity field which is more appropriate to model different flow situations. This is done by applying the CMM to each data set separately and then by merging the resulting estimates of transmissivity by means of some different algorithms. These algorithms could be standard averaging (arithmetic, geometric or harmonic mean, median value), or could be based on physical arguments.

Therefore, this paper is devoted to understand the effect of the parameter used to limit the dramatic effects of low hydraulic gradients and to the extension of the CMM for its application to multiple data sets. This is done by means of numerical tests, with different flow conditions, which permit to perform a critical analysis of the optimal parameter for the control of low hydraulic gradients and to assess the advantages and possible limitations of the application of CMM with multiple data sets.

In addition to the two aforementioned developments, aimed at improving our knowledge about the CMM, this work also illustrates the implementation of the CMM with well known free and open source software tools like Python and the USGS flopy and MODFLOW codes. This is done to share the implementation of the CMM with the scientific and professional community, in accordance with the concept of open science and with the aim of demonstrating and fostering practical application of inverse methods.

This paper is structured ad follows. Section 2 provides the theoretical background related to the CMM and to the approaches used to tackle the challenges posed by small hydraulic gradients and to improve the method's robustness by the use of multiple data-sets (tomographic approach). In Section 3 more technical details are given about the simulation settings used to test the CMM, the reference transmissivity field and the multiple data-set used for the tomographic approach are provided. Results are presented in Section 4, while Section 5 and Section 6 are devoted to discussion and conclusions, respectively.

\section{Methods}

It is assumed that a simulation model has to be developed and applied to predict the groundwater flow in a confined aquifer, under stationary conditions and by applying the hydraulic (2D) approximation (Bear, 2012).

The Comparison Model Method (CMM) is a direct method of inversion based on an auxiliary model, hereinafter called Comparison Model (CM). CM is based on a first guess of the transmissivity field $T^{(\mathrm{CM})}$ and shares the same source/sink terms and boundary conditions as the forward problem to be developed to simulate the dynamics of the confined aquifer. In practice, the transmissivity field is estimated by modifying $T^{(\mathrm{CM})}$ through the ratio of the hydraulic gradients computed by solving a forward problem (FP) for the CM and the reference hydraulic gradients:

$$
T^{(\mathrm{est})}=T^{(\mathrm{CM})} \frac{\left|\nabla h^{(\mathrm{CM})}\right|}{\left|\nabla h^{(\mathrm{ref})}\right|} .
$$

In real case applications, the difficulties in the definition of an initial guess of the field $T^{(\mathrm{CM})}$ and the errors on $h^{(\mathrm{ref})}$ can be handled by applying the method in an iterative fashion, that is

$$
T_{k}^{\text {(est) }}=T_{k-1}^{\text {(est) }} \frac{\left|\nabla h_{k-1}^{\text {(est) }}\right|}{\left|\nabla h^{(\text {ref })}\right|} \quad \text { with } \quad k \in\{1, \ldots, K\} .
$$

In (2) $k$ is the index corresponding to the $k$-th iteration of the method, $T_{k-1}^{(\text {est })}$ and $h_{k-1}^{(\text {est })}$ correspond to the transmissivity field of the CM at iteration $k-1$ and to the hydraulic head computed by solving the FP for that CM, respectively. In particular, for $k=0, T_{0}^{(\mathrm{est})}=T^{(\mathrm{CM})}$. Different criteria can be used to stop the CMM iterations, for example based on the reduction of a given error on hydraulic heads $h$ or simply based on the number of iterations. Hereinafter, as one of our aims is to compare the various settings given similar computational resources, a stopping criterion based on the number of iterations is considered.

Note that in (1), (2) and in the remainder of this paper, a node-to-node relationship is applied between the transmissivities and the head gradients, i.e. node $T$ values are updated by considering the corresponding local node head gradient.

However, other alternative approaches could be useful to limit some of the drawbacks of the crude node-to-node approach. For instance, a node-to-area relationship could be used to update node $T$ values by the mean hydraulic gradient 
over an area centered on the node.

\subsection{Dealing with small gradients}

By looking at equation (2) it is quite evident that for a trivial formulation of the CMM small values of $\left|\nabla h^{(\mathrm{ref})}\right|$ will entail numerical instabilities or ill-conditioning (Giudici et al., 2019). To cope with this issue, one possibility is to reformulate equation (2) by adding a tuning factor $\beta$

$$
T_{k}^{(\mathrm{est})}=T_{k-1}^{(\mathrm{est})}\left(1+\beta \frac{\left|\nabla h_{k-1}^{(\mathrm{est})}\right|-\left|\nabla h^{(\mathrm{ref})}\right|}{\left|\nabla h^{(\mathrm{ref})}\right|}\right) \text { with } k \in\{1, \ldots, K\} .
$$

as done for example in Vassena et al. (2012). The $\beta$ factor is selected according to the following rule

$$
\beta=\min \left\{\gamma\left|\nabla h^{(\mathrm{ref})}\right|, 1\right\} \quad \text { with } \quad \gamma \in \mathbb{R}_{+} .
$$

Notice that if $\gamma=1$, (3) reduces to the standard version (2). By means of (4) different values of the parameter $\gamma$, and therefore of the factor $\beta$, permit to fine tune the number of cells where the value of $T^{\text {(ref) }}$ will be computed using the standard formula (2) or with a correction to avoid the influence of small values of $\left|\nabla h^{(\text {ref })}\right|$. In the following the percentage of nodes where the correction is applied will be reported, because this seems more intuitive than the raw values of $\gamma$, but the two data are equivalent.

\subsection{Tomographic approach}

When hydraulic head measurements are made under different boundary/flow conditions, the corresponding datasets can be easily integrated into the CMM with a tomographic approach, as it is often done in many fields where inverse problems find application. This possibility was already explored by some of the earliest applications of the CMM (Scarascia and Ponzini, 1972; Ponzini and Crosta, 1988; Ponzini et al., 1989), but only in a one-dimensional (1D) framework. In this work the full potential of this approach is explored in $2 \mathrm{D}$, together with the implications of using different approaches to merge the information coming from each data-set.

The tomographic approach can be implemented as follows. Suppose some reference heads are available for a given number of data sets $L$. Then, at each iteration, $T$ can be estimated for each data set by (3), so that $L$ fields $T_{k, l}^{\text {(est) }}$, $l=1, \ldots, L$ are obtained. They are successively aggregated into a unique estimated field with the formula

$$
T_{k}^{(\mathrm{est})}=\mathcal{M}\left(T_{k, 1}^{(\mathrm{est})}, \ldots, T_{k, L}^{(\mathrm{est})}\right) \quad \text { with } \quad k \in\{1, \ldots, K\},
$$

where the function $\mathcal{M}$ is a merging operator.

Which merging operator $\mathcal{M}$ is best suited to stack the $T$ fields estimated for each data set is one of the research questions that will be tackled by this work. Hereinafter, six merging operators are considered, namely: arithmetic mean, geometric mean, harmonic mean, median, minimum correction and Darcy residuals. While the meaning of the first four operators is straightforward, the minimum correction and the Darcy residuals operators deserve more details.

With the minimum correction operator, at each point it is selected the value of $T_{k, l}^{(\text {est })}$ for which $\left|T_{k, l}^{(\text {est })}-T_{k-1}^{(\text {est })}\right|$ is minimum. This approach, although not physically based, should favor the more conservative estimates of $T$, i.e., those with the smallest variation between successive iterations, and therefore the solutions that are more stable through the CMM iterations.

With the Darcy residuals merging operator, a more physically based approach is chosen. One possible definition of Darcy residuals, inspired by the works of Kohn and Vogelius (1987) and Kohn and McKenney (1990), can be found in the supporting information of El-Rawy et al. (2014). Given a flow rate integrated over the aquifer thickness $Q$, the Darcy residuals can be defined at each point as a function $J=J(\sqrt{T})$ such that

$$
J=|Q / \sqrt{T}+\sqrt{T} \nabla h| .
$$

In (6), $Q$ and $\nabla h$, for each $l=1, \ldots, L$ are the values obtained from forward modelling with $T_{k-1, l}^{(\mathrm{est})}$, and $T=T_{k, l}^{(\mathrm{est})}$ is 

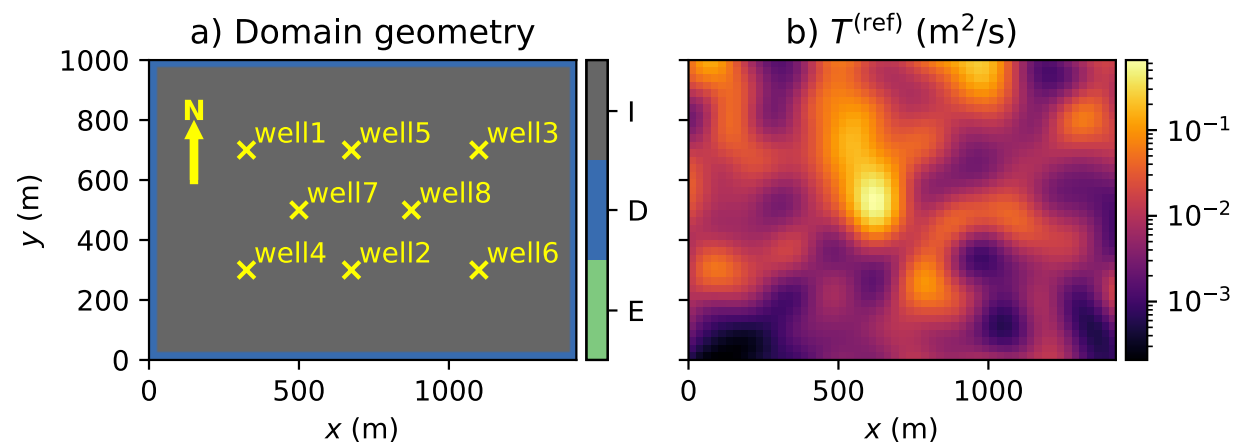

Figure 1: a) Geometry of the domain considered in this work: grey color is used for internal cells (code I), blue for fixed head boundary conditions (Dirichlet boundary conditions, code D) and green for external cells (code E, not used here but available if needed). The yellow crosses represent the locations of the pumping wells used for some of the following simulation settings. b) Reference $T$ field.

the CMM estimated transmissivity and iteration $k$.

The closer the function $J$ approaches to 0, the better Darcy's law is satisfied. Therefore, with the Darcy's residual criterion the merging operator will select, at each point, the value of $T_{k, l}^{(e s t)}, l=1, \ldots, L$ for which the function $J$ attains its minimum.

\section{Testing CMM}

This section provides more details about the flow simulation settings, the synthetic reference $T$ field, the input data used, the multiple-set of data for $h^{(\mathrm{ref})}$ and the criteria used to investigate the features of the CMM.

\subsection{Forward problem implementation and settings}

For testing in detail the various features of the CMM, the first requirement is the setting up of a flow simulation platform to solve the FP for a confined aquifer where the hydraulic approximation of two-dimensional (2D) flow is valid, under stationary conditions. To demonstrate and promote the usage of the CMM, in this work the well established USGS MODFLOW simulation platform, and in particular MODFLOW 6 (Hughes et al., 2017; Langevin et al., 2017; Provost et al., 2017; Langevin et al., 2019) is used. Note however that the CMM can be easily adapted to diverse simulation platforms, including ad hoc Fortran simulation codes (Scarascia and Ponzini, 1972; Ponzini and Lozej, 1982; Ponzini and Crosta, 1988; Ponzini et al., 1989; Vassena et al., 2012; Cattaneo et al., 2015), or the ParFlow simulation platform (Ashby and Falgout, 1996; Jones and Woodward, 2001; Kollet and Maxwell, 2006), as done by Comunian and Giudici (2018), or again by using the previous version MODFLOW-2005 during the preliminary part of this study.

An additional advantage of the MODFLOW platform is the availability of flopy (Bakker et al., 2016), a Python package that allows to easily write scripts to manage the execution and the access to the input/output files of MODFLOW and the related software. In this study, flopy (and Python) were extensively used to automate the implementation of the CMM, by running the FP simulations required by each CMM iteration, and to plot and analyse the results.

All the flow simulations are performed on a rectangular domain composed of $57 \times 40$ square cells of side $25 \mathrm{~m}$ (Fig. 1a) and one vertical layer. In Fig. 1a, grey color is used for internal cells (code I), blue is used for fixed head boundary conditions (Dirichlet boundary conditions, code D). It is of course possible to define domains of arbitrary shape by using the concept of external cells (here in green, code E), but for the sake of simplicity here a simple rectangular domain is used. The yellow crosses (Fig. 1a) are used to indicate the position of eight pumping wells that will be used in some of the simulation settings described hereinafter in diverse configurations. When activated, each of the wells will pump a volumetric flow rate of $25 \mathrm{~L} / \mathrm{s}$. Moreover, a diffuse recharge term of $250 \mathrm{~mm} / \mathrm{y}$ is applied to all the cells, in most tests.

\subsection{Reference and initial transmissivity field}

The reference transmissivity field considered throughout this work is the synthetic $T$ field represented in Fig. $1 \mathrm{~b}$. It is obtained with the Python package gstools (Müller and Schüler, 2019), that allows to easily generate random 
fields, and in particular a Gaussian covariance model generated with the algorithm proposed by Heße et al. (2014). The $T$ field shown in Fig. $1 \mathrm{~b}$ is obtained by

$$
T^{(\mathrm{ref})}\left(\mathbf{x}_{i}\right)=T_{0} \times 10^{Z\left(\mathbf{x}_{i}\right)},
$$

where $Z$ is a spatial random field with zero mean and variance 0.3 , following an isotropic Gaussian covariance with scale length 5 cells and estimated for each cell of coordinates $\mathbf{x}_{i}=\left(x_{i}, y_{i}\right) ; T_{0}$ is a constant value chosen to obtain a transmissivity that varies from about $2 \times 10^{-4} \mathrm{~m}^{2} / \mathrm{s}$ up to $0.66 \mathrm{~m}^{2} / \mathrm{s}$. In this work, the initial value of $T^{(\mathrm{CM})}$ was set homogeneous and equal to the arithmetic mean of $T^{(\mathrm{ref})}$.

\subsection{Boundary conditions and source terms to generate multiple data sets}

In this study, two groups of multiple data sets are considered to test the tomographic approach described in Section 2.2. The first group of data sets is created by imposing linearly-varying fixed-head boundary conditions along four flow directions, all rotated by $45^{\circ}$ increments. This results in four $h$ fields, with main flow direction towards south, south-west, west and north-west (in the following these flow conditions will be labeled with the corresponding letters $\mathrm{S}, \mathrm{S}-\mathrm{W}, \mathrm{W}, \mathrm{N}-\mathrm{W})$. The resulting reference heads for the aforementioned linearly-varying boundary conditions are represented in Fig. 2. Hereinafter, this group of data-sets will be referred as ROT (data-sets group with rotated main flow directions). Clearly, changes of $45^{\circ}$ of the main flow directions are not expected under seasonal variations on real world aquifers. Nevertheless, here this setting was considered to demonstrate some of the benefits of the tomographic approach under ideal conditions. A test made by using more realistic ranges of variability $\left(10^{\circ}\right)$ was performed and reported in Appendix A.1.

Notice that ROT for null source terms satisfy the conditions for univalent $\sigma$-harmonic mappings (Alessandrini and Nesi, 2001).

The second group of data sets considered is created by running flow simulations with a uniform, prescribed-head boundary condition (Fig. 1a) and by alternatively turning on one of the wells defined in the same Fig. 1a. In this way, eight data sets of $h^{(\mathrm{ref})}$ are obtained, corresponding to eight different flow conditions, each induced by a single pumping well. Hereinafter, this group of data-sets will be referred as WEL (data-sets group created with diverse configurations of pumping wells).

The computation of the hydraulic gradients is performed with the numpy function gradient, using second order accurate central differences.

\subsection{Testing CMM robustness}

To assess the robustness of the method against noisy reference data, the CMM was run by using one or more $h^{(\text {ref) }}$ data-sets affected by a noise having a uniform Gaussian spatial structure with scale length 5 cells, and a standard deviation ranging from $1 \mathrm{~cm}$ to $10 \mathrm{~cm}$; the comparisons also included a setting with noiseless $h^{(\mathrm{ref})}$.

In the case of noisy data, it was also tested the importance of the use of multiple data sets to improve the method's robustness. In particular, the impact of noisy data with variable standard deviation was assessed by using a multiple data set of four head conditions (main flow directions along directions $\mathrm{S}, \mathrm{W}, \mathrm{SN}$ and $\mathrm{NW}$ ), a multiple data set of two head conditions (main flow directions along directions $\mathrm{S}$ and $\mathrm{W}$ ) and a unique data set (main flow direction along $\mathrm{S}$ ).

Note that for a real case application, only sparse measurements of $h^{(\mathrm{ref})}$ are available, possibly affected by noise, and one should interpolate them, for example using kriging, at all the nodes of the domain before applying the CMM. In this work, to avoid the possible subjectivity inherent in the interpolation, e.g., for the selection of the variogram model used in the kriging interpolation, it was preferred to directly add a structured noise to the noiseless $h^{(\text {ref })}$ data, which can mimic the effect of interpolation errors.

\subsection{Evaluation criteria}

The results obtained with the diverse inversion setting explored for the CMM are evaluated using a number of criteria to provide a comparison with the results obtained for $T^{(\mathrm{ref})}$. A first group of indicators are the statistics computed at all the $N$ nodes where a $T$ value is estimated, which are defined for each iteration step $k$ as:

$$
\lambda_{k}=\frac{1}{N} \sum_{i=1}^{N}\left[\log \left(T^{(\mathrm{ref})}\left(\mathbf{x}_{i}\right)\right)-\log \left(T_{k}^{(\mathrm{est})}\left(\mathbf{x}_{i}\right)\right)\right],
$$


a) south (S)

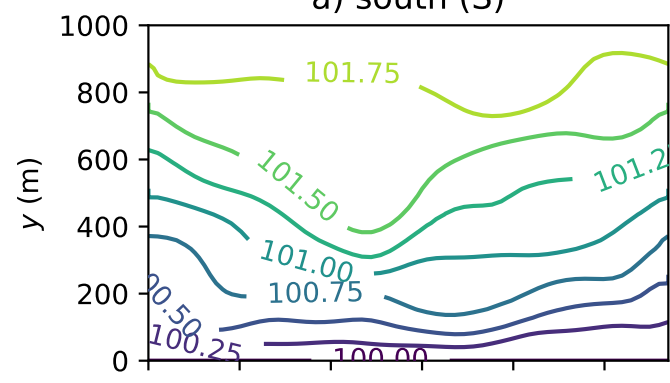

c) south-west (SW)

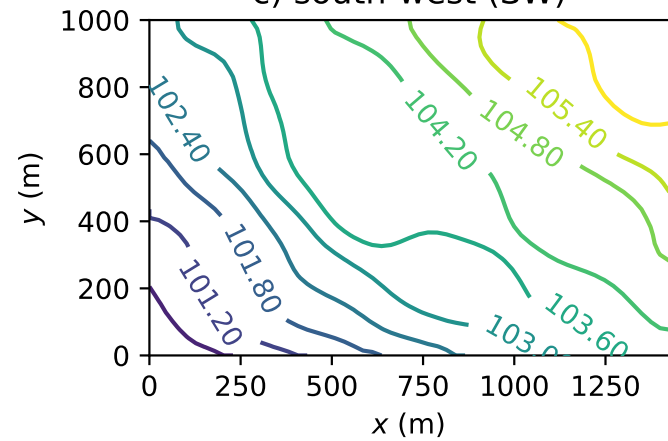

b) west (W)

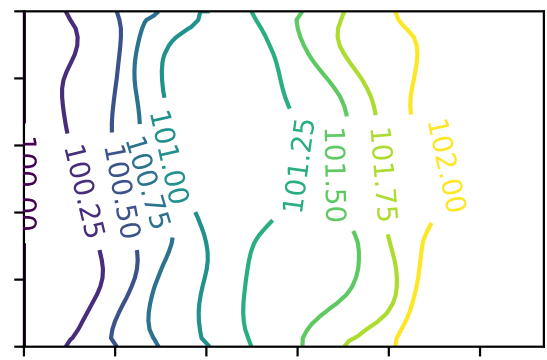

d) north-west (NW)

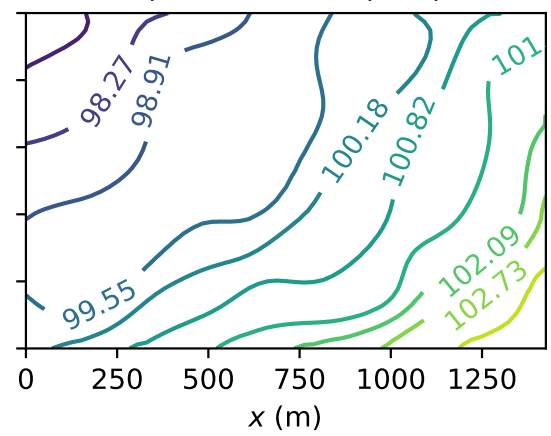

Figure 2: The reference $h$ fields for the first group of multiple data sets (ROT) with main flow along the a) south direction $(\mathrm{S})$; b) west direction (W); c) south-west direction (S-W) and d) north-west direction (N-W).

Table 1

Summary of the settings used to run the CMM in this work

\begin{tabular}{|c|c|c|c|c|}
\hline \multirow[b]{3}{*}{ setting } & \multicolumn{4}{|c|}{ test aim } \\
\hline & \multirow{2}{*}{$\gamma$ selection } & \multirow{2}{*}{$\mathcal{M}$ selection } & \multicolumn{2}{|c|}{ robustness test } \\
\hline & & & noiseless data & noisy data \\
\hline corrected $\nabla h^{(\mathrm{ref})}(\%)$ & $100,60,30,15,0$ & 15 & 15 & 15 \\
\hline merging operator $\mathcal{M}$ & arithmetic mean & all $^{a}$ & geometric mean & geometric mean \\
\hline recharge $(\mathrm{m} / \mathrm{s})$ & $7.9 \times 10^{-9}$ & 0 & 0 or $7.9 \times 10^{-9}$ & 0.0 \\
\hline active wells (ID) $^{b}$ & 1,2, and 3 & none & none or one well ${ }^{c}$ & none \\
\hline noise $\sigma(\mathrm{cm})$ & 0 & 0 & 0 & from 0.1 to 10 \\
\hline data-sets group & ROT & ROT & ROT or WEL & ROT \\
\hline specific data-set & $\mathrm{S}$ & S, SW, W, NW & $\mathrm{S}, \mathrm{SW}, \mathrm{W}, \mathrm{NW} /$ single well $^{d}$ & S, SW, W, NW \\
\hline \multicolumn{5}{|c|}{$\begin{array}{l}\text { a arithmetic mean, geometric mean, harmonic mean, median, Darcy's residuals, minimum correction } \\
{ }^{b} \text { wells are numbered from } 1 \text { to } 8 \text {; their location is illustrated in Fig. } 1 \text { a } \\
{ }^{c} \text { only when the data-set group WEL is used, the well related to the corresponding data set is activated } \\
{ }^{d} h^{\text {(ref) }} \text { data-set corresponding to one of the eight wells activated one by one }\end{array}$} \\
\hline$\lambda_{k}^{2}=\frac{1}{N} \sum_{i=1}^{N}[\mathrm{lo}$ & $\left.T^{(\mathrm{ref})}\left(\mathbf{x}_{i}\right)\right)-\log$ & $\left.\left(T_{k}^{(\mathrm{est})}\left(\mathbf{x}_{i}\right)\right)\right]^{2}$ & & \\
\hline
\end{tabular}

with a straightforward meaning. In particular, $\lambda^{2}$ represents the mean squared error (MSE). In addition to these three statistics, other indicators were also computed for a given iteration step $k$ : maps of the head anomaly $A_{k}=h^{\text {(ref) }}-h_{k}^{\text {(est) }}$, that is the difference between the reference hydraulic heads and the hydraulic heads corresponding to the estimated $T$ 
field; the mean value of $\left|A_{k}\right|$ over the considered domain; maps of $\log \left(T^{(\text {ref })}\right)-\log \left(T_{k}^{(\text {est })}\right)$.

\section{Results}

Table 1 lists the settings used to run the CMM according to the aim of each test, namely: selection of the $\gamma$ parameter; selection of the $\mathcal{M}$ operator; tests of robustness with respect to errors on head data.

\subsection{Selecting the parameter $\gamma$}

To explore the impact of the choice of the parameter $\gamma$, the CMM was run with six different choices of this parameter. Actually, the Python implementation of the CMM presented in this work allows to directly set the percentage of cells where the correction defined by (4) applies rather than defining explicitly the value of $\gamma$. In fact, in general, the choice of the parameter $\gamma$ depends on the considered data set, and setting up only the percentage of the modified cells is much more intuitive. Therefore, once defined the percentage of affected cells, the value of the parameter $\gamma$ is automatically computed from $h^{\text {(ref) }}$.

The set up of the FP was deliberately kept simple, by considering only one data set (main flow directed along the south direction, Fig. 2a), a recharge term and three wells activated, well1, well2 and well3 (see the column " $\gamma$ selection" in Table 1 for more details).

Fig. 3 represents the $T$ fields obtained after $10 \mathrm{CMM}$ iterations for a percentage of corrected data of $100 \%, 60 \%$, $30 \%, 15 \%$ and $0 \%$. Visual inspection of the $T$ fields reveals that when $100 \%$ of the gradients are corrected, then a big part of the information concerning the transmissivity contrasts is lost, resulting in a very smooth $T$ field (Fig. 3b). Results obtained by correcting 60\%,30\%, 15\% and $0 \%$ of the cell gradients are comparable (Fig. 3b, c, d, e and f). Nevertheless, low percentages of corrected data provide better estimates of the high $T$ peak observed in the center of the domain. In addition, a non-null percentage of corrected cells allows to avoid the unrealistic $T$ values obtained for very small values of the gradient, for example in the cells south of well2, or in the high $T$ region south of well 3 (Fig. 3f).

The same results are represented in Fig. 4 in terms of $\lambda^{2}$ for all the iterations. To illustrate the comparison tools adopted in this work, some additional plots are reported here, for the particular case of a percentage of corrected data equal to $15 \%$. The first group of these plots represents the trend of the errors $\lambda$ and $\lambda^{2}$ (Fig. 5a and b) and the mean value of the anomaly $|A|$ (Fig. 5c). The two other plots represent a map of the anomaly $|A|$ (Fig. 6a) and a map of $\log \left(T^{(\text {ref })}\right)-\log \left(T_{5}^{\text {(ref) }}\right)$ (Fig. 6b), where for both plots the CMM iteration step 5 was selected only for illustration purposes. Note that plots like Fig. 5 are drawn for each CMM settings, and plots like Fig. 6 are drawn for each iteration of CMM settings. Clearly, here only summary plots are reported.

On the basis of these results, which will be discussed more in detail in Section 5, it has been decided to set the percentage of cells where $\beta<1$ to $15 \%$ for all the tests presented in the following sections.

\subsection{Selecting the merging operator}

No wells were activated and no recharge was applied, and the four different data sets obtained by imposing linear varying Dirichlet boundary conditions with a main direction of flow oriented toward four directions described in Section 3.1 and Fig. 2 were used simultaneously as multiple data sets (data sets group ROT). With these settings, 10 CMM iterations were performed by using one of the six merging operators proposed in Section 2.2 at a time. The resulting $T$ fields are reported in Fig. 7 for the arithmetic mean (Fig. 7a), the geometric mean (Fig. 7b), the harmonic mean (Fig. 7c), the median (Fig. 7d), the Darcy residuals (Fig. 7e) and the minimum correction (Fig. 7f) merging operators. The same six merging operators are compared in terms of $\lambda^{2}$ (Fig. 8).

Overall, by considering both the visual inspection of the $T$ fields (Fig. 7) and how $\lambda^{2}$ evolves with the iterations (Fig. 8), apart from the minimum correction, the results obtained with the other merging operators are comparable. Given the good performances of the geometric mean as merging operator (for a detailed discussion see Section 5) and some theoretical considerations concerning effective parameters (Matheron, 1967), the tests described in the following sections are performed by using this merging operator.

\subsection{Usefulness of the tomographic approach}

To verify the usefulness of the tomographic approach, tests were performed by using both a noiseless $h^{(\mathrm{ref})}$ and an $h^{(\mathrm{ref})}$ affected by a gradually growing structured noise. The details of the two tests are provided in the following sections. 

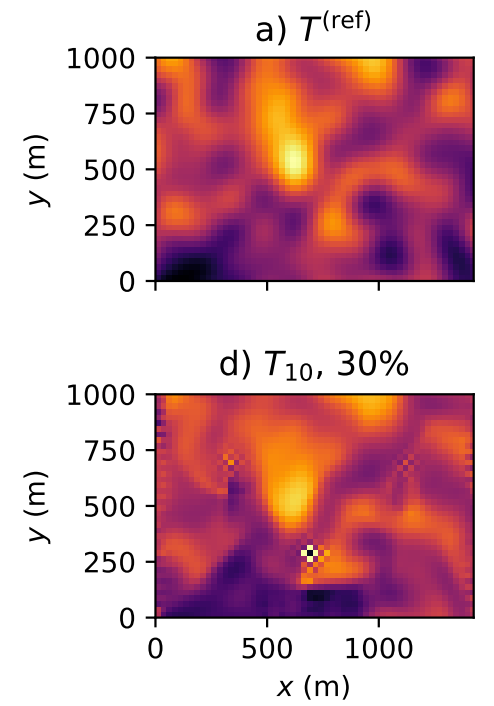

b) $T_{10}, 100 \%$

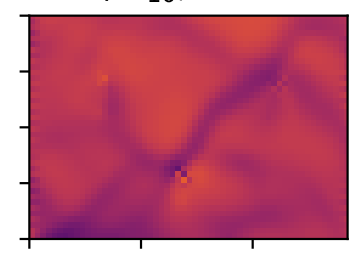

e) $T_{10}, 15 \%$

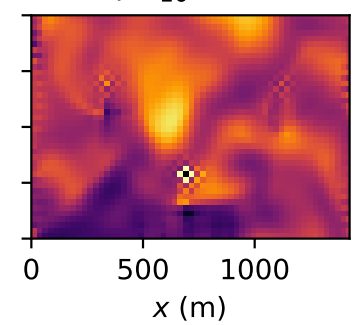

c) $T_{10}, 60 \%$

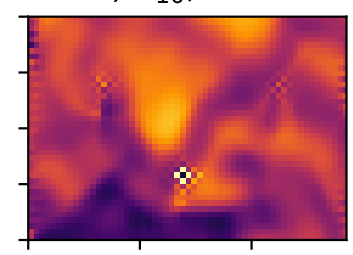

f) $T_{10}, 0 \%$

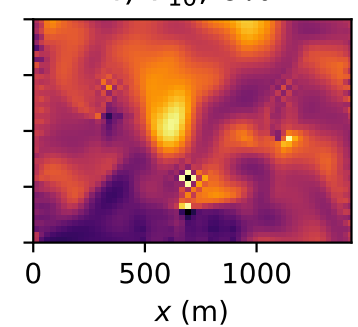

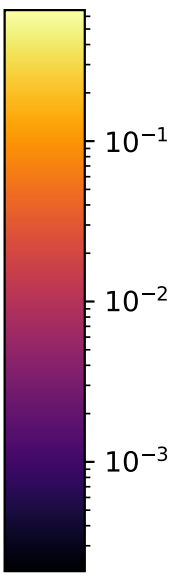

Figure 3: Comparing the resulting $T$ fields for different choices of the parameter $\gamma$. a) Reference $T$ field; b), c), d), e), f) $T$ computed after 10 iteration steps of the CMM for diverse values of the parameter $\gamma$. The corresponding percentage of corrected data reported close to each label from b) to f). Units are $\mathrm{m}^{2} / \mathrm{s}$.

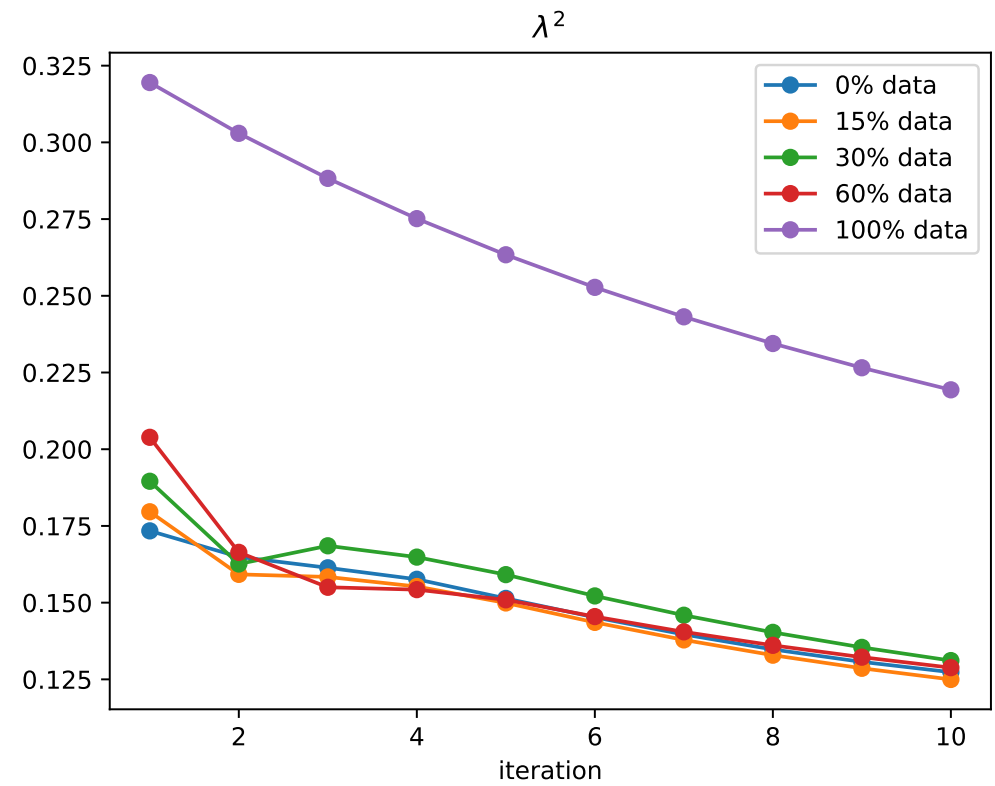

Figure 4: $\lambda^{2}$ vs CMM iteration for different values of the parameter $\gamma$. The legend reports the percentage of domain cells where $\left|\nabla h^{(\text {ref })}\right|$ was corrected for the corresponding value of $\gamma$.

\subsubsection{Noiseless reference $h$}

This test was performed by using two different groups of data-sets. In the first group of data-sets, multiple reference $h$ fields were obtained by changing some linear varying Dirichlet boundary conditions, as explained in Section 2.2 and Fig. 2 (ROT). In the second group of data-sets, multiple reference $h$ fields were obtained by activating alternatively one and only one pumping well among the eight wells represented in Fig. 1a, and by considering a constant $h$ value on the boundaries of the domain (WEL).

By using the first group of data-sets (ROT), four settings of the CMM were run using a growing number of datasets, ranging from one data-set (flow direction towards south, that is data-set S), two (S and SW), three (S, SW and 

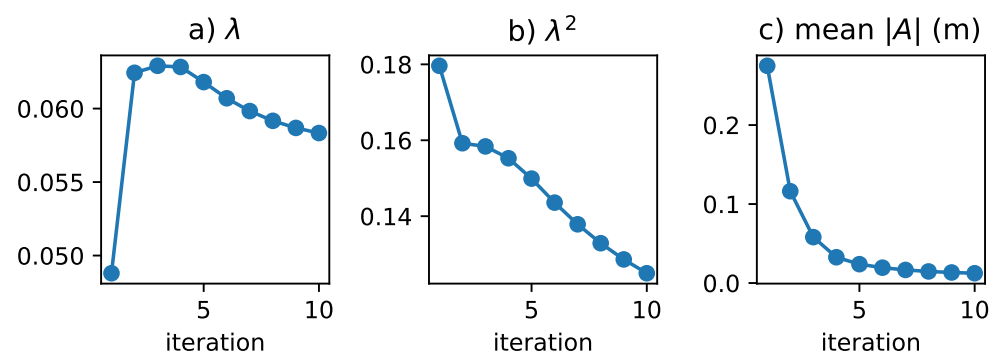

Figure 5: Diagnostic plots for the case when $15 \%$ of small $\left|\nabla h^{(\text {ref })}\right|$ are corrected (tests performed for the selection of $\gamma$ ): a) error $\lambda$, b) $\lambda^{2}$ and c) mean value of $|A|$.
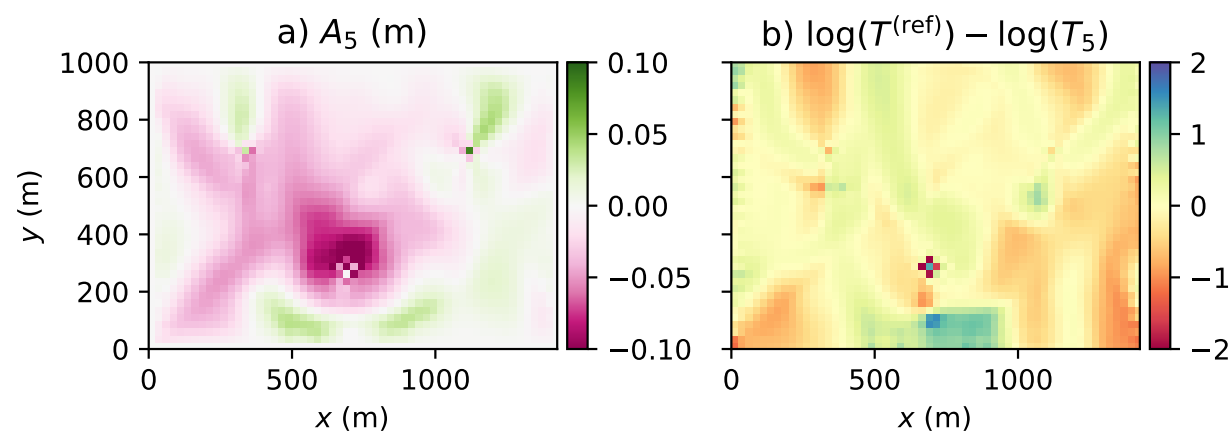

Figure 6: Maps of anomaly on $h$ and error on $T$ for CMM iteration 5.

W) and finally four data-sets (S, SW, W, and NW). From the visual inspection of the $T$ fields it is already evident that there is an improvement of the reproduced reference transmissivity field (Fig. 9), and that the greatest differences are introduced by using at least two data-sets. The same observations can be made on the comparison of the four CMM runs in terms of $\lambda^{2}$ (Fig. 10a), where at the 10th iteration the value of $\lambda^{2}$ for the settings where all the four data-sets are used (S, SW, W, and NW) is about $1 / 5$ of the $\lambda^{2}$ that corresponds to the usage of one data-set only (S).

The second group of data sets (WEL) was used to run eight settings of the CMM with an approach similar to that adopted for ROT: the first CMM setting makes use of only one $h^{(\mathrm{ref})}$ data set, corresponding to the conditions were only one pumping well is activated (well1); the second CMM setting makes use of two $h^{(\mathrm{ref})}$ data sets, one corresponding to well1 activated, the other corresponding to well2 activated; and so on up to the last CMM setting, which makes use of eight $h^{(\text {ref })}$ data-sets, each one corresponding to the flow conditions where only one of the available pumping wells is activated. In analogy to the first group of data-sets, the values of $\lambda^{2}$ are plotted against the number of CMM iterations (Fig. 10b). For this second group similar improvements when adding an additional $h^{(\text {ref })}$ can be noticed like for the first group. However, $\lambda^{2}$ values are overall greater than those recorded for the ROT data-set group (Fig. 10).

\subsubsection{Noisy reference $h$}

One important part of this work was to asses the robustness of the CMM in case of noisy data. To check this, a noise with a standard deviation growing from $1 \mathrm{~cm}$ to $10 \mathrm{~cm}$ and having the same scale length of the reference $T$ field (but generated with a different random seed) was added to all the $h^{(\text {ref })}$ data-sets corresponding to the flow conditions illustrated in Section 2.2 (Fig. 2). The results of this test are shown in Fig. 11 in terms of $\lambda^{2}$. As the differences in the improvement from $\lambda^{2}$ computed with tree data-sets (, $\mathrm{SW}$, and $\left.\mathrm{W}\right)$ and four data-sets $(\mathrm{S}, \mathrm{SW}, \mathrm{W}$, and NW) were minor, for the sake of brevity only the results obtained by using four data-sets (S, W, SW, and NW; Fig. 11a), two data-sets (S, and W; Fig. 11b), and one data-set (S only; Fig. 11c) are shown here. In Fig. 11 the three subplots share the same axes. This allows to clearly illustrate the importance of using multiple sets of data: in fact, even if for a noise of about $10 \mathrm{~cm}$ the $\lambda^{2}$ starts to diverge after 10 iterations (Fig. 11a) when using four data-sets, the values of $\lambda^{2}$ always remains much smaller than the values attained when two data-sets (Fig. 11b) or one data-set (Fig. 11c) are used. 
a) arithmetic mean
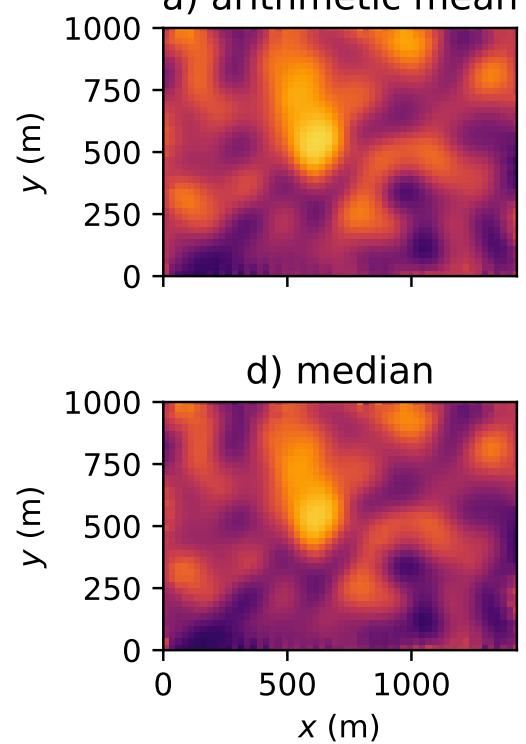

b) geometric mean

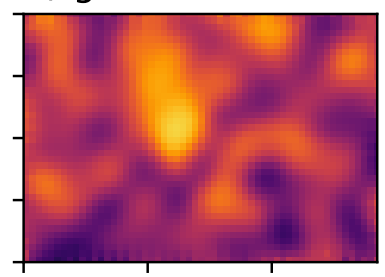

e) Darcy residuals

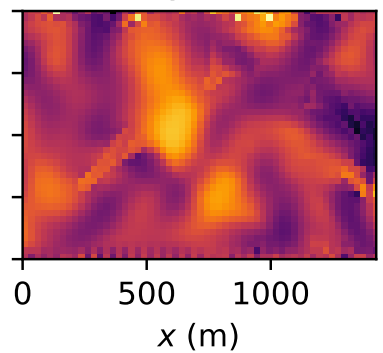

c) harmonic mean

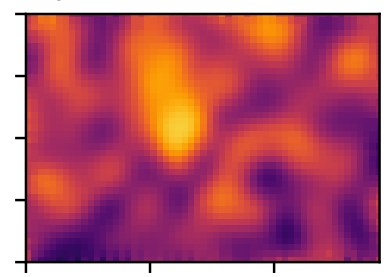

f) minimum correction

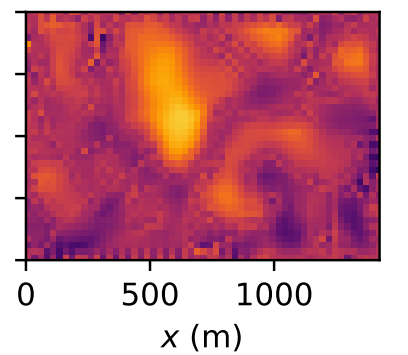

Figure 7: $T$ fields obtained with different merging operators after $10 \mathrm{CMM}$ iterations: a) arithmetic mean; b) geometric mean; c) harmonic mean; d) median; e) Darcy residuals and f) minimum correction. Units $a^{2} \mathrm{~m}^{2} / \mathrm{s}$.

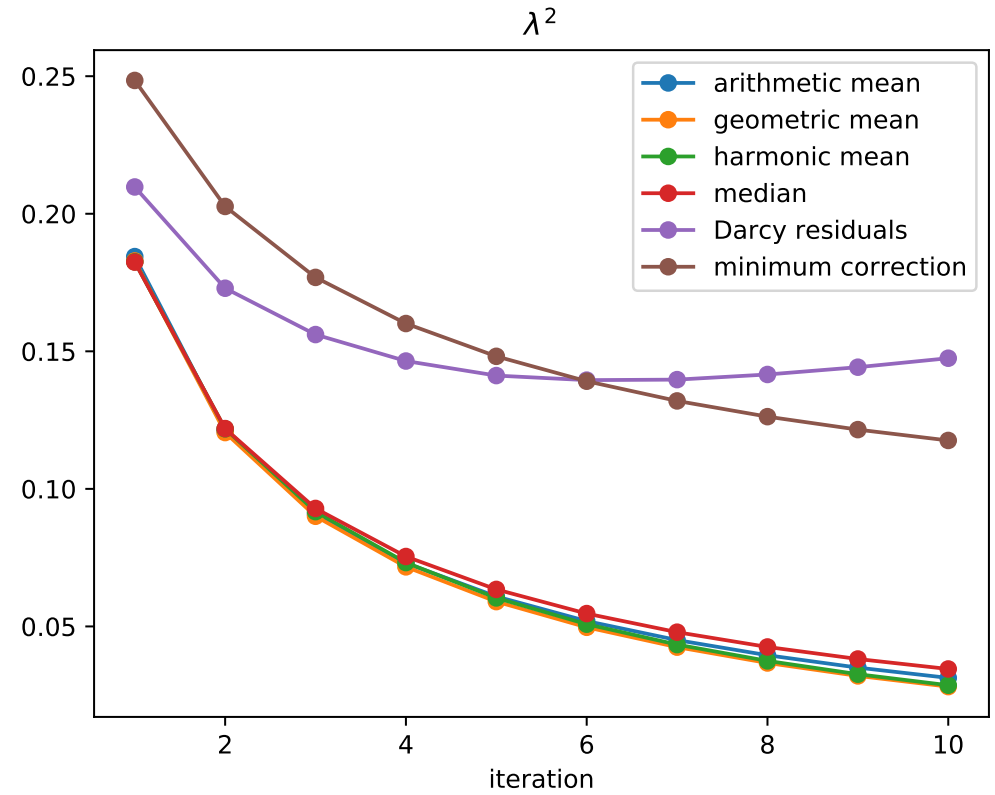

Figure 8: $\lambda^{2}$ vs CMM iteration for different merging operators.

\section{Discussion}

One of the research questions investigated here was related to the selection of the best values for the parameter $\gamma$, a parameter that allows to select which proportion of $\nabla h^{(\text {ref })}$ data-set should be corrected to avoid numerical instabilities. The results presented in Fig. 3 and Fig. 4 show that correcting a small percentage of the small $\nabla h^{(\text {ref })}$ should be sufficient to prevent the numerical instabilities that would lead to unrealistic values of $T$. It is quite clear that when $100 \%$ of the gradients are corrected, a big part of the information contained in the data-set is lost (Fig. 4b). Instead, without any correction, the estimated $T$ field presents some very high values, for example few cells south of well1 and well3. 


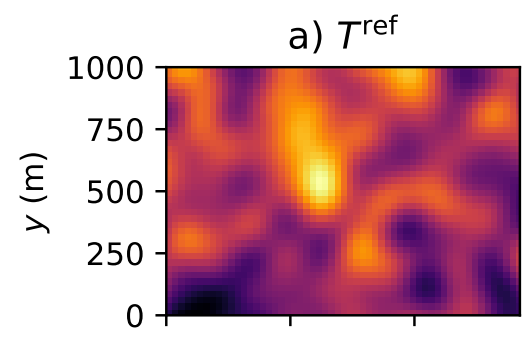

b) $T_{10}-\mathrm{S}$

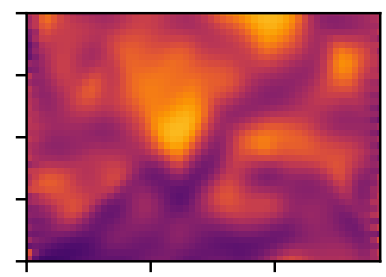

d) $T_{10}-\mathrm{S}, \mathrm{SW}, \mathrm{W}$

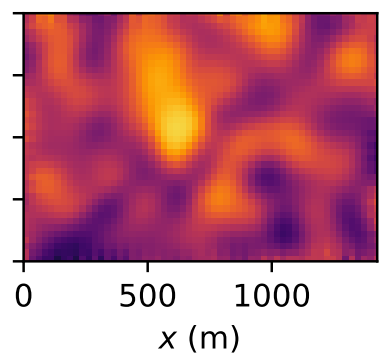

c) $T_{10}-\mathrm{S}, \mathrm{SW}$

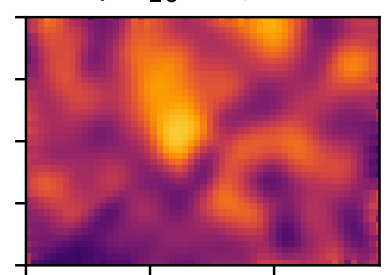

e) $T_{10}-\mathrm{S}, \mathrm{SW}, \mathrm{W}, \mathrm{NW}$

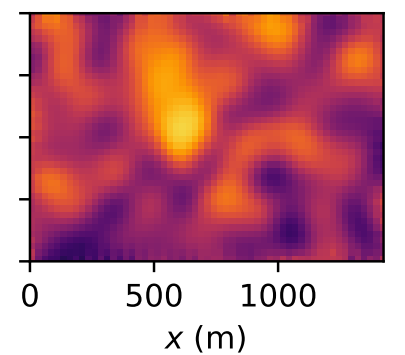

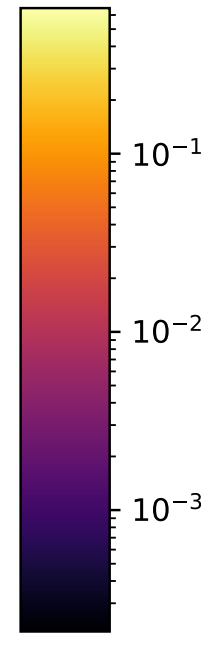

Figure 9: $T$ fields obtained considering a different number of data sets from the ROT group: a) reference $T$; b) one data set used, flow direction $\mathrm{S} ; \mathrm{c}$ ) two data set used, flow direction $\mathrm{S}$ and $\mathrm{SW} ; \mathrm{d}$ ) three data set used, flow direction $\mathrm{S}, \mathrm{SW}$ and $\mathrm{W} ;$ e) four data set used, flow direction S, SW, W and NW. Units are $\mathrm{m}^{2} / \mathrm{s}$.

a) $\lambda^{2}-$ ROT

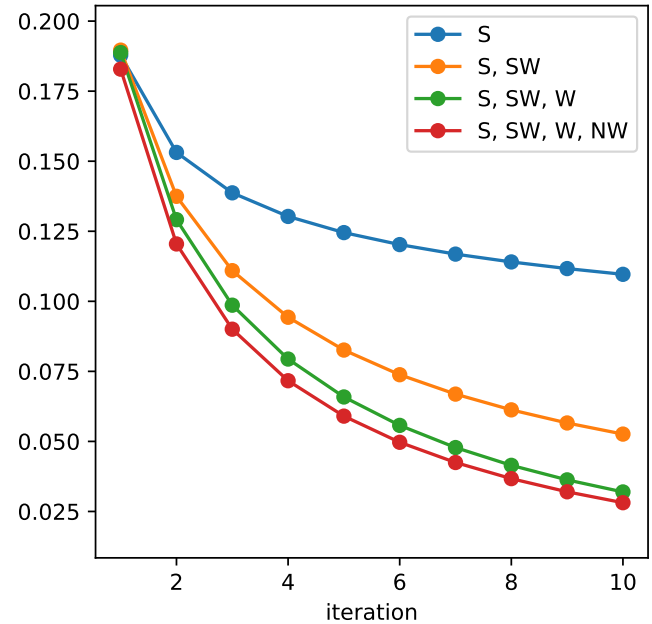

b) $\lambda^{2}-$ WEL

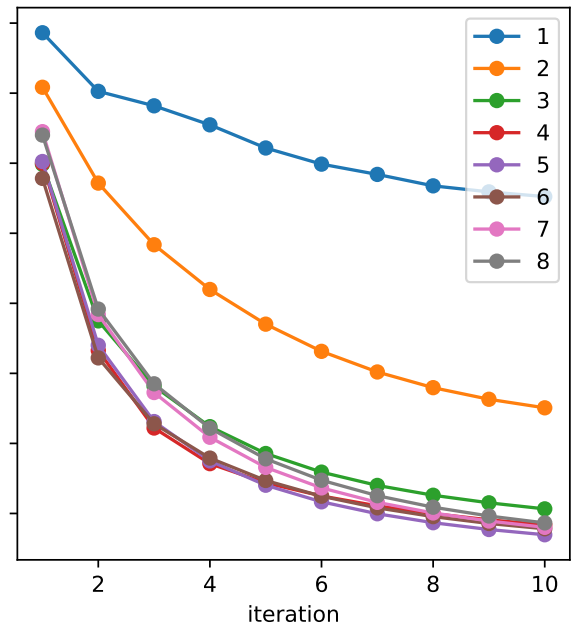

Figure 10: $\lambda^{2}$ vs CMM iteration for increasing number of data sets considered: a) four data sets in total (ROT) corresponding to different flow directions, starting from only one direction (blue line) and adding up to four directions considered simultaneously (red line); b) eight data sets in total (WEL), where for each data set only one well is activated, and uses incrementally from one (blue line) to eight (grey line) data sets.

While in all the previous works (see i.e., Vassena et al., 2012) it was required to explicitly define a value of the factor $\beta$, here a simple and practical procedure, that allows to select a value for $\gamma$ as a function of the number of corrected gradient data, is implemented.

Another important aspect systematically investigated in this work is the selection of the best merging operator $\mathcal{M}$ to pool estimates of $T$ coming from diverse data-sets. Among the six methods investigated, five methods (arithmetic mean, geometric mean, harmonic mean, median and the more physically based Darcy residuals) provided comparable results, while another tested method (minimum correction) creates some artifacts for intermediate values of transmissivity (Fig. 7) and performs worst in terms of $\lambda^{2}$ (Fig. 8). Given the very small differences both in terms of visual 
a) $\lambda^{2}$ - data sets S, W, SW and NW

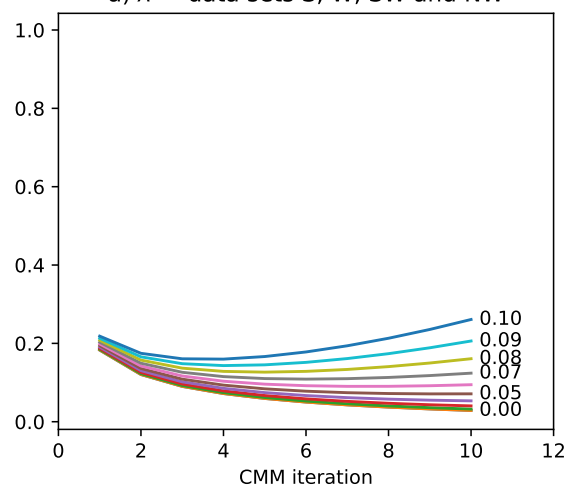

b) $\lambda^{2}$ - data sets $S$ and $W$

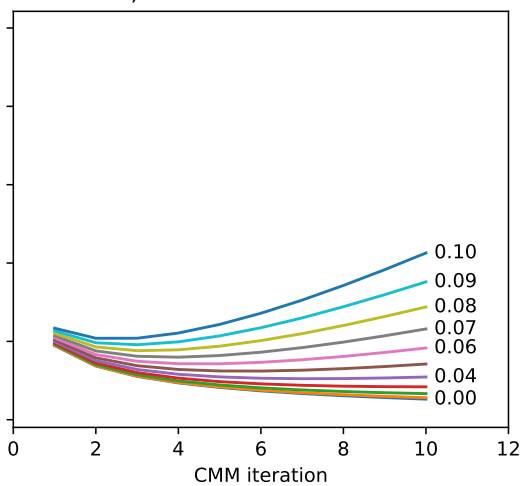

c) $\lambda^{2}-$ data set $\mathrm{S}$

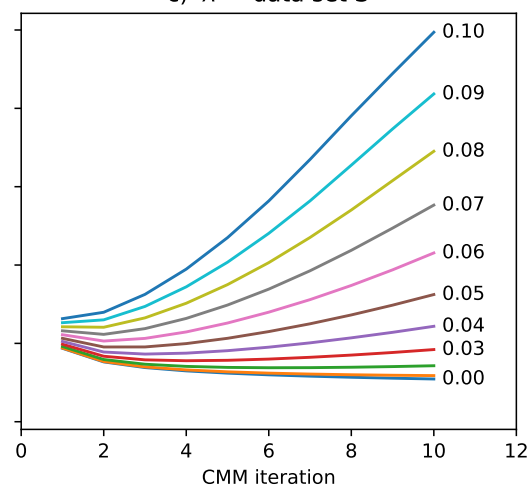

Figure 11: $\lambda^{2}$ vs CMM iteration for increasing number of data sets and increasing noise on reference $h$ for the group ROT: a) four data sets, flow along S, W, SW and NW directions; b) two data sets, flow along S and W direction; c) one data set, flow along $\mathrm{S}$ direction. The labels reported at the end of each line represent the standard deviation of noise on $h$ ( $\mathrm{m}$ ), which varies from $0.01 \mathrm{~m}$ (blue lines) to $0.1 \mathrm{~m}$ (cyan lines) with steps of $0.01 \mathrm{~m}$. Some labels are omitted due to overlapping with other labels.

inspection of the estimated $T$ fields and in terms of $\lambda^{2}$, one could select as merging operator the geometric mean also on the basis of some theoretical findings related to effective hydraulic parameters (Matheron, 1967).

To demonstrate the usefulness of the tomographic approach, two tests were performed using noiseless $h^{(\mathrm{ref})}$ data and one test was performed using noisy data. All the three tests demonstrate the usefulness of multiple data-sets. The first two tests were conducted on two groups of data-sets, one based on different flow conditions created by using linearly varying Dirichlet boundary conditions (ROT, Fig. 2), the other based on different flow conditions created by activating one by one eight different pumping wells (WEL). For the former, Fig. 9 and Fig. 10a show that by using more than one data-set both reduces $\lambda^{2}$ and improves the reconstruction of the contrasts of $T$ (Fig. 9). Actually, already by adding one additional data-set ( $\mathrm{S}$ and SW) allows to drastically improve the results, and in particular $\lambda^{2}$ (Fig. 10a). The differences between the results obtained with three and four data-sets are relatively small and hardly detectable by the visual inspection of the $T$ fields, although still noticeable in the $\lambda^{2}$ plots (Fig. 10).

Similar results were obtained for the WEL group of multiple data-set, obtained by activating alternatively one by one the pumping wells. In this case, however, the trend in the improvements of the results is less obvious than the one obtained by setting flow condition along four different directions as done for the previous test. Here, the additional information provided when one well alone is pumping might depend on its location within the domain and the corresponding $T^{(\mathrm{ref})}$ value. In particular, one can notice a good improvement on $\lambda^{2}$ when the data-set corresponding to well2 is added (orange line, Fig. 10b). However, adding a third data-set (corresponding to well3, green line, Fig. 10b) only provides small improvements on $\lambda^{2}$. Similar observations can be made concerning the addition of data-sets related to other wells.

It is also interesting to compare the results obtained with the first group of data-sets (ROT, four main flow directions, S, SW, W, and NW) against the second group (WEL, eight wells turned on with pumping one by one): the improvements obtained by adding few data-sets corresponding to the first group are much more marked than for the second case because, as expected, changing the boundary conditions in such a way that the $h$ field over the whole domain is affected is much more effective than changing flow conditions only locally by activating one pumping well. This is also demonstrated when multiple data sets having smaller differences in terms of main flow directions are used (see appendix A.1 for details).

The last test performed in this study verified the robustness of the method in presence of noisy data (Fig. 11c). When only one data-set is used, $T_{k}^{(\text {est })}$ converges with a growing number of iterations $k$ only for noise with a standard deviation on $h^{(\text {ref })}$ below $2 \mathrm{~cm}$. However, by just adding another data-set (Fig. 11b) the divergence of $\lambda^{2}$ remains relatively limited, even with a noise with a standard deviation of $5 \mathrm{~cm}$. When all the four data-sets are used there is a further improvement: when the standard deviation on the noise is greater than $6 \mathrm{~cm}, \lambda^{2}$ starts to diverge, but its values remain nevertheless below $0.2 \mathrm{~m}^{4} / \mathrm{s}^{2}$.

As a final comment, it is important to stress that the MSE $\lambda^{2}$ obtained with a single, noise-free data set is very 
close to the one which corresponds to the application of the CMM with four sets of data with noisy hydraulic heads, up to a standard deviation of $10 \mathrm{~cm}$. The absolute value of the standard error is not significant per se, but should be compared, for instance, with the total variation of the $h^{(\text {ref })}$ field, which in this example is of the order of few meters. Therefore, in these numerical tests, the application of the CMM to multiple sets of data provides results comparable to those of the standard CMM with a single set of data at least for a "signal-to-noise-ratio", estimated as the ratio between the standard deviation of noise and the total variation of $h^{(\mathrm{ref})}$, approximately equal to 0.05 .

\section{Conclusions}

This work allowed to explore more in detail many features of the Comparison Model Method by using a synthetic but realistic and challenging reference transmissivity field $T^{(\mathrm{ref})}$ (Fig.1b). The first feature explored provides guidelines for the selection of the parameter used to correct very low values of the gradient of $h^{(\mathrm{ref})}$ : it is proposed a straightforward procedure to select the parameter based on the percentage of domain cells where the gradient is corrected, and it was demonstrated that already with small percentages there is an improvement on the results. Another aspect explored in this work concerns the selection of a reliable merging operator when multiple data-sets are used for the solution of the inverse problem with a tomographic approach. Among the method tested, apart from the minimum correction method, all the other merging operators provided comparable results, and one can for example select the geometric mean operator as also supported by some theoretical background (Matheron, 1967).

Besides aspects related to the merging operator, in the framework of a tomographic approach one can also test the impact of the usage of a diverse number of data-sets, both noiseless and noisy. Here we tested this aspect by using two groups of data-sets, and in all the cases it was demonstrated that using more data-sets allows to better identify the $T$ field. Of course, depending on the nature and the variability of the available data-sets, one can reveal a different amount of information on the parameters of the investigated transmissivity field. For example, the group of data-sets representing diverse main flow directions allowed to identify the $T^{(\mathrm{ref})}$ better than the group of data-sets obtained by turning on/off one pumping well.

Another important point demonstrated by this work is the robustness of the CMM in the presence of noisy data, and in particular the usefulness of the tomographic approach. In fact, as the application of the CMM requires an interpolation step, measurements errors, the interpolation algorithm and the values of the algorithm's parameters (e.g., the semivariogram model for interpolation with kriging) can noticeably affect the final results. Therefore, the robustness of the CMM was verified by running the method with reference $h$ data affected by a noise with a spatial structure and a growing standard deviation. When four data-sets are used, $\lambda^{2}$ remains moderate even for a noise with a standard deviation greater than $5 \mathrm{~cm}$. One could observe that this error would be relatively small for a typical hydraulic head measurement network. Nevertheless, one should also consider that in the CMM, all the reference data used for the inversion were considered as affected by noise, and this for each one of the data-sets.

One of the aim of this work was also to foster the usage of direct inversion methods as an alternative to other more computational demanding inversion paradigms, and to provide for this aim ready to use tools to demonstrate its applicability in diverse settings. Section "Computer code availability" provides the link for the download of almost all the CMM related implementation used to obtain the results presented in this study.

Based on the achievement of this work, and in particular the ones related to the tomographic approach, one could extend the works done by Comunian and Giudici (2018) by using multiple-data sets to avoid the strong influence of a unique flow condition when used as a secondary variable in a direct-sampling simulation framework (Mariethoz et al., 2010).

Also, additional reference $T^{(\mathrm{ref})}$ fields having a diverse spatial nature could be used; nevertheless, the authors believe that the synthetic test already represents a realistic and challenging heterogeneous field.

Concerning the CMM, other future research direction could include tests made by changing the aquifer resolution or strategies for a better selection of the initial value for the $T^{(\mathrm{CM})}$, the latter being another critical aspect of the methodology.

Clearly, many research questions remain open in the realm of model calibration in groundwater hydrology, from the perspective of both direct and indirect inversion techniques. This work explores some improvements and provides a tool to foster the usage of the CMM, a direct inversion method that in some cases could represent a frugal alternative (or a syde by syde companion) to the widely used indirect approach. 


\section{Computer code availability}

An implementation of the CMM based on flopy and MODFLOW 6, called cmmpy (version 0.1.3) is provided, together with the accompanying scripts and parameter files, at the following link:

https://bitbucket.org/alecomunian/cmmpy with a GNU General Public License. The corresponding Python package is also included in the Python Package Index (PyPI), and it can be easily installed with the command pip install cmmpy. The documentation is available at the link https://cmmpy.readthedocs.io.

\section{Acknowledgements}

This work was partially performed in the framework of the project "Geosciences for society: resources and their evolution" supported by the Italian Ministry of University and Research (MUR) through the fund 'Dipartimenti di Eccellenza 2018-2022'.

\section{References}

Alessandrini, G., Nesi, V., 2001. Univalent $\sigma$-harmonic mappings. Archive for Rational Mechanics and Analysis 158, 155-171. doi:10.1007/ PL00004242.

Ashby, S.F., Falgout, R.D., 1996. A parallel multigrid preconditioned conjugate gradient algorithm for groundwater flow simulations. Nuclear Science and Engineering 124, 145-159. doi:10.13182/NSE96-A24230.

Bakker, M., Post, V., Langevin, C.D., Hughes, J.D., White, J.T., Starn, J.J., Fienen, M.N., 2016. Scripting MODFLOW model development using Python and FloPy. Groundwater 54, 733-739. doi:10.1111/gwat. 12413.

Baratelli, F., Flipo, N., Moatar, F., 2016. Estimation of stream-aquifer exchanges at regional scale using a distributed model: Sensitivity to in-stream water level fluctuations, riverbed elevation and roughness. Journal of Hydrology 542, 686 - 703. doi:10.1016/j.jhydrol.2016.09.041.

Bear, J., 2012. Hydraulics of Groundwater. Dover Books on Engineering, Dover Publications.

Brouwer, G., Fokker, P., Wilschut, F., Zijl, W., 2008. A direct inverse model to determine permeability fields from pressure and flow rate measurements. Mathematical Geosciences 40, 907-920. doi:10.1007/s11004-008-9183-3.

Carrera, J., Alcolea, A., Medina, A., Hidalgo, J., Slooten, L.J., 2005. Inverse problem in hydrogeology. Hydrogeology journal 13, 206-222. doi:10.1007/s10040-004-0404-7.

Cattaneo, L., Comunian, A., de Filippis, G., Giudici, M., Vassena, C., 2015. Modeling groundwater flow in heterogeneous porous media with YAGMod. Computation 4, 2. doi:10.3390/computation4010002.

Comunian, A., Giudici, M., 2018. Hybrid inversion method to estimate hydraulic transmissivity by combining multiple-point statistics and a direct inversion method. Mathematical Geosciences doi:10.1007/s11004-018-9727-0.

Dagasan, Y., Juda, P., Renard, P., 2020. Using generative adversarial networks as a fast forward operator for hydrogeological inverse problems. Groundwater 58, 938-950. doi:https://doi.org/10.1111/gwat.13005.

De Filippis, G., Foglia, L., Giudici, M., Mehl, S., Margiotta, S., Negri, S.L., 2017. Effects of different boundary conditions on the simulation of groundwater flow in a multi-layered coastal aquifer system (Taranto Gulf, southern Italy). Hydrogeology Journal 25, 2123-2138. doi:10.1007/ s10040-017-1589- $\mathrm{x}$.

De Filippis, G., Giudici, M., Margiotta, S., Negri, S., 2016. Conceptualization and characterization of a coastal multi-layered aquifer system in the Taranto Gulf (southern Italy). Environmental Earth Sciences 75, 686. doi:10.1007/s12665-016-5507-7.

De Filippis, G., Margiotta, S., Branca, C., Negri, S.L., 2019. A Modelling Approach for Assessing the Hydrogeological Equilibrium of the Karst, Coastal Aquifer of the Salento Peninsula (Southeastern Italy): Evaluating the Effects of a MAR Facility for Wastewater Reuse. Geofluids 2019, 5714535. doi:10.1155/2019/5714535.

Doherty, J., 2015. Calibration and Uncertainty Analysis for Complex Environmental Models. Watermark Numerical Computing, Brisbane, Australia.

El-Rawy, M., Batelaan, O., Zijl, W., 2014. Simple hydraulic conductivity estimation by the kalman filtered double constraint method. Groundwater , 401-413doi:10.1111/gwat.12217.

Franssen, H.H., Alcolea, A., Riva, M., Bakr, M., Van der Wiel, N., Stauffer, F., Guadagnini, A., 2009. A comparison of seven methods for the inverse modelling of groundwater flow. application to the characterisation of well catchments. Advances in Water Resources 32, 851-872. doi:10.1016/j.advwatres.2009.02.011.

Giudici, M., 2003. Some problems for the application of inverse techniques to environmental modeling. Contemporary mathematics 333, 89-98.

Giudici, M., Baratelli, F., Cattaneo, L., Comunian, A., Filippis, G.D., Durante, C., Giacobbo, F., Inzoli, S., Mele, M., Vassena, C., 2019. conceptual framework for discrete inverse problems in geophysics. arXiv:1901.07937.

Giudici, M., Ginn, T.R., Vassena, C., Haeri, H., Foglia, L., 2008. A critical review of the properties of forward and inverse problems in groundwater hydrology, in: ModelCARE, IAHS Press. pp. 240-244.

Giudici, M., Margiotta, S., Mazzone, F., Negri, S., Vassena, C., 2012. Modelling hydrostratigraphy and groundwater flow of a fractured and karst aquifer in a Mediterranean basin (Salento peninsula, southeastern Italy). Environmental Earth Sciences 67, 1891-1907. doi:10.1007/ s12665-012-1631-1.

Giudici, M., Morossi, G., Parravicini, G., Ponzini, G., 1995. A new method for the identification of distributed transmissivities. Water Resources Research 31, 1969-1988. doi:10.1029/95WR01205.

Giudici, M., Vassena, C., 2006. dsm.f90: A computer code for the solution of an inverse problem of ground water hydrology by the differential system method. Computers \& Geosciences 32, 1709 - 1719. doi:10.1016/j . cageo.2006.03.008. 
Giudici, M., Vassena, C., 2008. Spectral analysis of the balance equation of ground water hydrology. Transport in Porous Media 72, 171-178. doi:10.1007/s11242-007-9142-3.

Heße, F., Prykhodko, V., Schlüter, S., Attinger, S., 2014. Generating random fields with a truncated power-law variogram: A comparison of several numerical methods. Environmental Modelling \& Software 55, 32 - 48. doi:10.1016/j . envsoft. 2014.01.013.

Hill, M.C., Tiedeman, C.R., 2006. Effective groundwater model calibration: with analysis of data, sensitivities, predictions, and uncertainty. John Wiley \& Sons.

Hughes, J., Langevin, C., Banta, E., 2017. Documentation for the MODFLOW 6 framework. chapter A57. p. 40. doi:10.3133/tm6A57.

Jones, J.E., Woodward, C.S., 2001. Newton-Krylov-multigrid solvers for large-scale, highly heterogeneous, variably saturated flow problems. Advances in Water Resources 24, 763 - 774. doi:10.1016/S0309-1708(00)00075-0.

Kitanidis, P.K., 2007. On stochastic inverse modeling. Geophysical Monograph-American Geophysical Union 171, 19. doi:10.1029/171GM04.

Kohn, R., McKenney, A., 1990. Numerical implementation of a variational method for electrical impedance tomography. Inverse Problems 6 , 389-414. doi:10.1088/0266-5611/6/3/009.

Kohn, R.V., Vogelius, M., 1987. Relaxation of a variational method for impedance computed tomography. Communications on Pure and Applied Mathematics 40, 745-777. doi:10.1002/cpa.3160400605.

Kollet, S.J., Maxwell, R.M., 2006. Integrated surface-groundwater flow modeling: A free-surface overland flow boundary condition in a parallel groundwater flow model. Advances in Water Resources 29, 945 - 958. doi:10.1016/j . advwatres . 2005.08.006.

Langevin, C., Hughes, J., Banta, E., Provost, A., Niswonger, R., Panday, S., 2019. MODFLOW 6 Modular Hydrologic Model version 6.1.0. doi:10.5066/F76Q1VQV.

Langevin, C.D., Hughes, J.D., Banta, E.R., Niswonger, R.G., Panday, S., Provost, A.M., 2017. Documentation for the MODFLOW 6 Groundwater Flow Model. Technical Report. U. S. Geological Survey. Reston, VA.

Lesnic, D., 2010. The comparison model method for determining the flexural rigidity of a beam. Journal of Inverse and Ill-posed Problems 18, 577-590. doi:10.1515/jiip.2010.026.

Linde, N., Renard, P., Mukerji, T., Caers, J., 2015. Geological realism in hydrogeological and geophysical inverse modeling: A review. Advances in Water Resources 86, Part A, 86 - 101. doi:10.1016/j . advwatres .2015.09.019.

Mariethoz, G., Renard, P., Straubhaar, J., 2010. The direct sampling method to perform multiple-point geostatistical simulations. Water Resour. Res. 46, W11536. doi:10.1029/2008WR007621.

de Marsily, G., Delhomme, J.P., Coudrain-Ribstein, A., Lavenue, A.M., 2000. Four decades of inverse problems in hydrogeology. Geological Society of America Special Papers 348, 1-17. doi:10.1130/0-8137-2348-5.1.

Matheron, G., 1967. Eléments pour une Théorie des Milieux Poreux, Masson, Paris. Masson, Paris.

Müller, S., Schüler, L., 2019. GeoStat-Framework/GSTools: Reverberating Red v1.1.0. doi:10.5281/zenodo.3468230.

Neuman, S.P., 1973. Calibration of distributed parameter groundwater flow models viewed as a multiple-objective decision process under uncertainty. Water Resources Research 9, 1006-1021. doi:10.1029/WR009i004p01006.

Parravicini, G., Giudici, M., Morossi, G., Ponzini, G., 1995. Minimal a priori assignment in a direct method for determining phenomenological coefficients uniquely. Inverse Problems 11,611. doi:10.1088/0266-5611/11/3/009.

Poeter, E.P., Hill, M.C., 1999. Ucode, a computer code for universal inverse modeling. Computers \& Geosciences 25 , 457 - 462. doi:https : //doi.org/10.1016/S0098-3004(98)00149-6.

Ponzini, G., Crosta, G., 1988. The comparison model method: A new arithmetic approach to the discrete inverse problem of groundwater hydrology. Transport in Porous Media 3, 415-436. doi:10.1007/BF00233178.

Ponzini, G., Crosta, G., Giudici, M., 1989. Identification of thermal conductivities by temperature gradient profiles; one-dimensional steady flow. Geophysics 54, 643-653. doi:10.1190/1.1442691.

Ponzini, G., Lozej, A., 1982. Identification of aquifer transmissivities: The comparison model method. Water Resources Research 18, 597-622. doi:10.1029/WR018i003p00597.

Provost, A., Langevin, C., Hughes, J., 2017. Documentation for the "XT3D" option in the Node Property Flow (NPF) Package of MODFLOW 6. chapter A56. p. 40. doi:10.3133/tm6A56.

Scarascia, S., Ponzini, G., 1972. An approximate solution of the inverse problem in hydraulics. L'Energia Elettrica 49, 518-531.

Vassena, C., Durante, C., Giudici, M., Ponzini, G., 2008. The importance of observations on fluxes to constrain ground water model calibration. Physics and Chemistry of the Earth, Parts A/B/C 33, 1105 - 1110. doi:10.1016/j.pce.2008.01.004.

Vassena, C., Rienzner, M., Ponzini, G., Giudici, M., Gandolfi, C., Durante, C., Agostani, D., 2012. Modeling water resources of a highly irrigated alluvial plain (Italy): calibrating soil and groundwater models. Hydrogeology Journal 20, 449-467. doi:10.1007/s10040-011-0822-2.

Vesselinov, V.V., Harp, D.R., 2012. Adaptive hybrid optimization strategy for calibration and parameter estimation of physical process models. Computers \& Geosciences 49, 10 - 20. doi:https : //doi.org/10.1016/j.cageo.2012.05.027.

Zhou, H., Gómez-Hernández, J.J., Li, L., 2014. Inverse methods in hydrogeology: Evolution and recent trends. Advances in Water Resources 63, 22 - 37. doi:10.1016/j . advwatres.2013.10.014.

\section{A. Further remarks}

\section{A.1. Tomographic approach in a natural setting}

In section 4.3.1 the usefulness of the tomographic approach was demonstrated by using multiple data-sets, and in particular by using multiple reference $h$ fields obtained by changing some linearly-varying Dirichlet boundary conditions to obtains settings with the main flow direction oriented along $\mathrm{S}, \mathrm{SW}, \mathrm{W}$ and $\mathrm{NW}$. These differences in orientation of $45^{\circ}$ were useful to enlighten some features of tomographic approach. However, such a high difference in orientation 
a) $0^{\circ}$

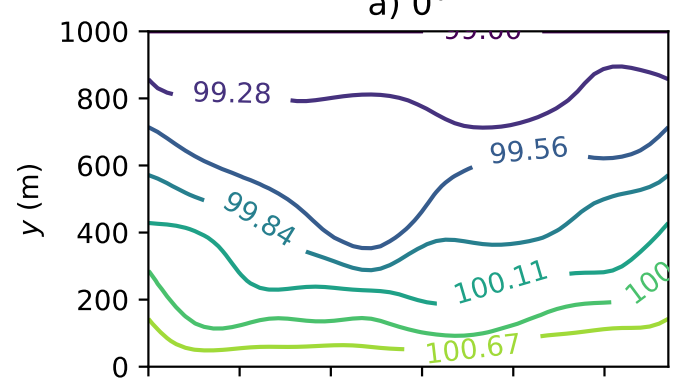

c) $20^{\circ}$

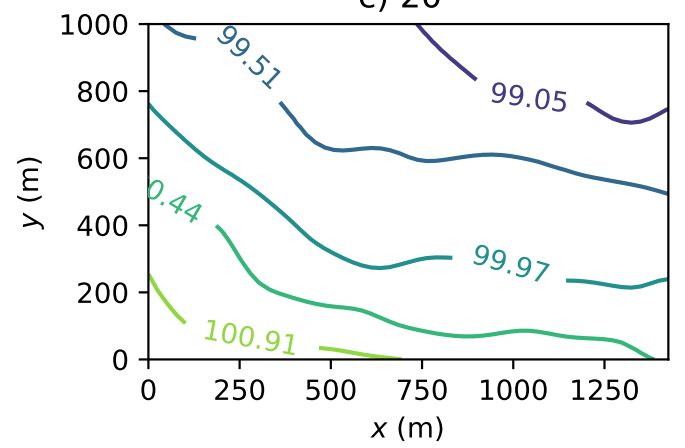

b) $10^{\circ}$

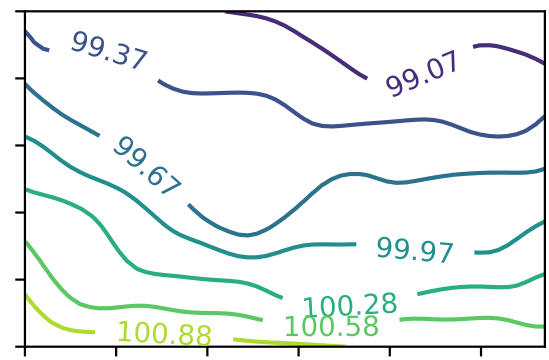

d) $30^{\circ}$

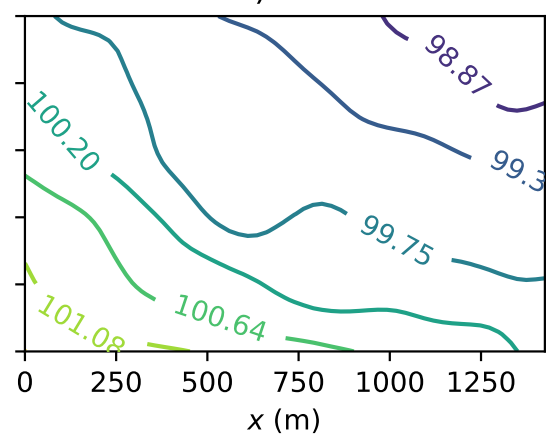

Figure 12: Reference $h$ fields used to test the tomographic approach with multiple data with having closer main flow directions, ranging from $0^{\circ}, 10^{\circ}, 20^{\circ}, 30^{\circ}$ north.

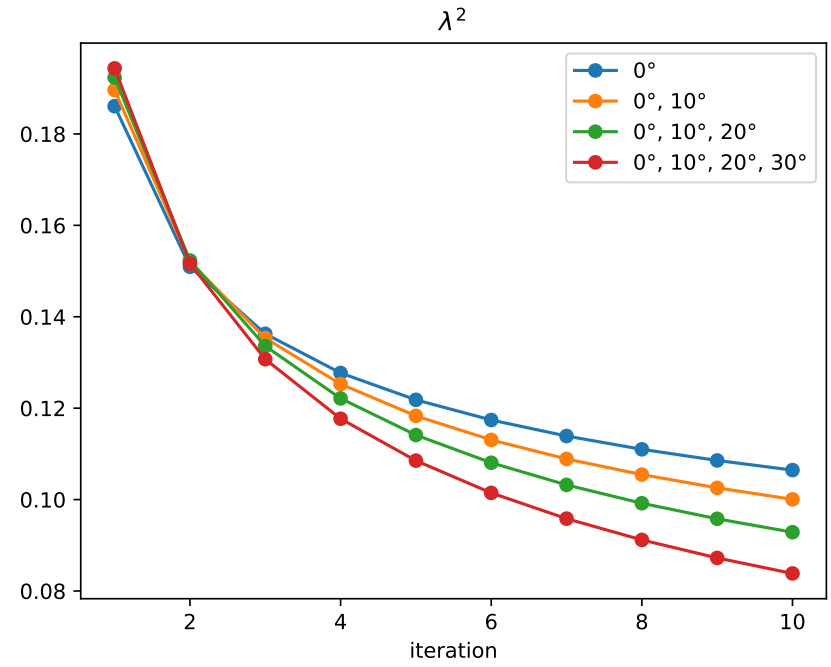

Figure 13: $\lambda^{2}$ vs CMM iterations for increasing number of data sets considered. Each data set corresponding to different flow directions, starting from only one direction $\left(0^{\circ}\right.$, blue line) and adding up to four directions considered simultaneously $\left(0^{\circ}, 10^{\circ}, 20^{\circ}\right.$ and $30^{\circ}$; red line $)$.

would be hardly attained under natural conditions in an aquifer. Therefore, in this appendix we test the usefulness of the tomographic approach with a more realistic group of data, with orientations of the main flow directions that differ by $10^{\circ}$ each other (Fig. 12) and a gradient of $1 \mathrm{~mm} / \mathrm{m}$. The results illustrated in Fig. 13 clearly show the improvements in terms of $\lambda^{2}$ even when multiple data having only small changes in the main flow directions are used. Similar consideration can be drawn by comparing the obtained $T$ fields against the reference ones (results not shown here). 
a) $\lambda^{2}$

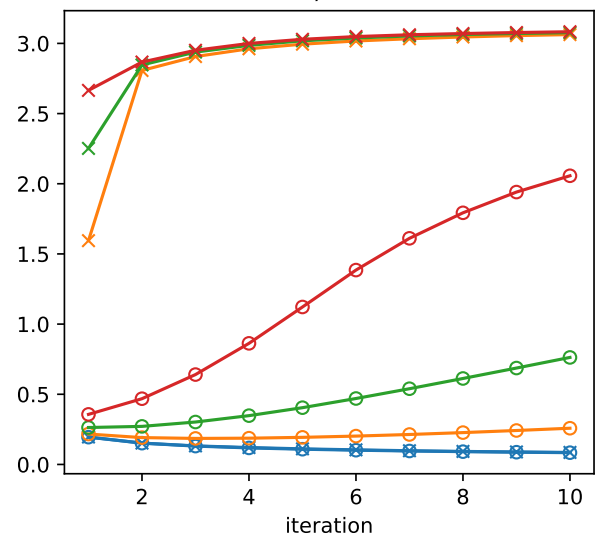

b) MAE on $h$

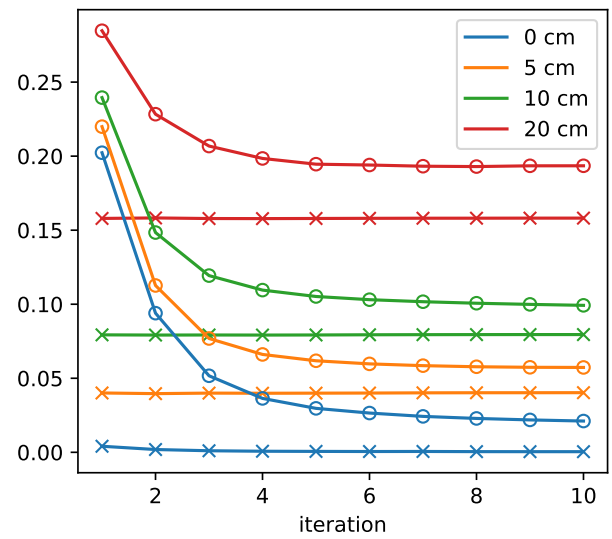

Figure 14: a) $\lambda^{2}$ vs CMM iterations for different values of standard deviation error on the $h$ data sets (cyan $0 \mathrm{~cm}$, yellow $5 \mathrm{~cm}$, green $10 \mathrm{~cm}$, and red $20 \mathrm{~cm})$ and for different values of average gradient on $h(1 \mathrm{~cm} / \mathrm{m} \mathrm{cross} \mathrm{markers,} 2 \mathrm{~cm} / \mathrm{m}$ circle markers). b) Mean absolute error (MAE) on the reference $h$ fields vs iterations, with the same notation of part a). (For plot b) only the MAE on the data set with main flow direction along $0^{\circ}$ is shown).

\section{A.2. Effects of errors on $h$ for different head gradients}

Further tests were performed to verify the behaviour of the CMM for diverse magnitudes of the input head gradient in response to a growing error on the head field. In particular, the example illustrated in Appendix A.1 is expanded by considering groups of data sets with two different average $h$ gradients, $1 \mathrm{~mm} / \mathrm{m} 2 \mathrm{~mm} / \mathrm{m}$. Moreover, in addition to the noiseless head condition, the tests were also performed by adding to each group of data sets a structured noise with standard deviation of $5 \mathrm{~cm}, 10 \mathrm{~cm}$ and $20 \mathrm{~cm}$. The results of these tests are summarized in Fig. 14 .

This test is important to enlighten the different impact of the noise on different discrete hydraulic gradients, as discussed for example by Giudici (2003), Remark 4.3. Concerning this topic, see also pp11-12 of Giudici et al. (2019).

In Fig. 14a) is represented the RMSE ( $\lambda^{2}$ ) for different values of standard deviations of the noise added to the noiseless data and for different values of the average gradient of the reference $h$ data sets. The different colours used in the plots correspond to the different standard deviations, ranging from $0 \mathrm{~cm}$ (cyan), $5 \mathrm{~cm}$ (yellow), $10 \mathrm{~cm}$ (green) and $20 \mathrm{~cm}$ (red). The different markers correspond to the average gradients of the input data sets: crosses are used for the $1 \mathrm{~mm} / \mathrm{m}$ gradient, while circles are used for the $2 \mathrm{~mm} / \mathrm{m}$ gradient.

When noiseless data are used, regardless of the average magnitude of the gradient, the behaviour of the curve for an increasing number of iterations is almost the same, with a rather good convergence trend (see the overlapping blue lines with circle and cross markers). Instead, when the standard deviation of the error on the $h$ data set rises, the responses of the data set with different gradients is quite different, with the smallest gradient $(1 \mathrm{~mm} / \mathrm{m}$, cross markers) reacting much more than the highest gradient $(2 \mathrm{~mm} / \mathrm{m}$, circle markers $)$. After a couple of iterations, in fact, the curves corresponding to the smallest gradient rise quickly to a value of $\lambda^{2}$ close to 3 , and are almost indistinguishable despite the diverse standard deviation errors. Differently, for the highest gradient, the dependence on the standard deviation errors is more differentiated and lower values of $\lambda^{2}$ are attained.

With the same notation introduced for Fig. 14a), in Fig. 14b) are represented the mean absolute errors (MAE) on $h$ as a function of the iteration number. As in the test illustrated in Appendix A.1, in this and in the following tests four $h$ data sets with the same average gradient and main flow direction ranging from $0^{\circ}$ to $30^{\circ}$ north are used for each couple of gradient/error parameter sets. However, only the MAE corresponding to the data set oriented along $0^{\circ}$ is shown here, because the MAE computed for the other directions yield very similar results.

Fig. 14b) shows that, when compared with the MAE corresponding to the highest gradient (circle markers), the MAE obtained with the smallest gradient converges towards a value few $\mathrm{cm}$ below the corresponding standard deviation error (cross markers). Differently, the MAE on the highest gradient starts with values above $20 \mathrm{~cm}$ in the first iteration, and then converges towards values very close to the standard deviation error after a few of iterations. In all the test described up to this point, whenever a noise is added to the reference $h$, the noise had a spatial structure (structured noise).

An additional test was performed with the same settings of the test summarized in Fig. 14 but using an unstructured 
a) $\lambda^{2}$

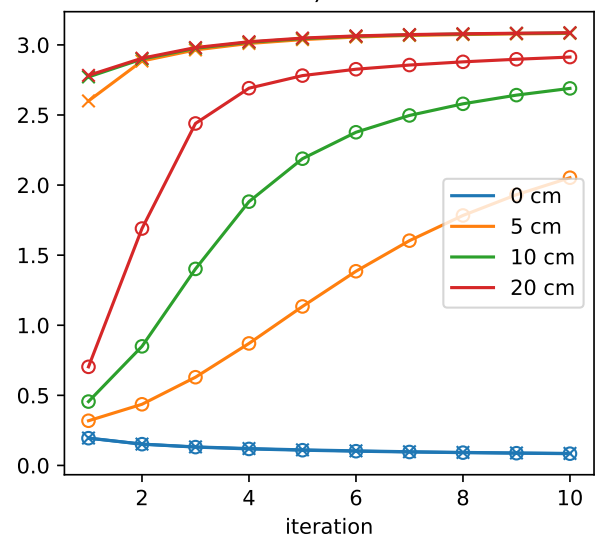

b) MAE on $h$

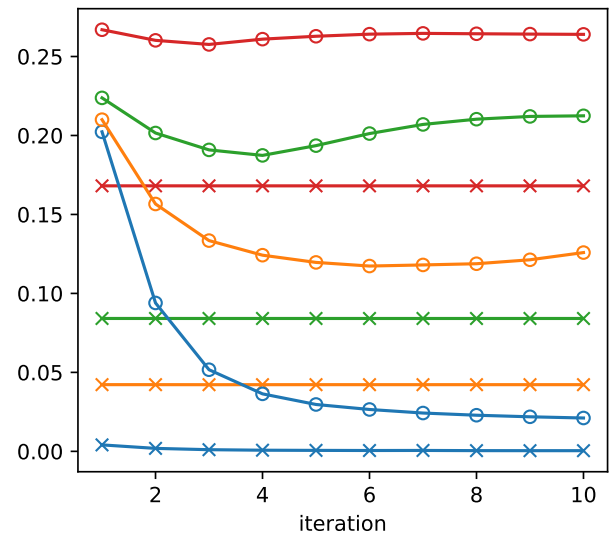

Figure 15: Same results shown in Fig. 14, but for an uncorrelated noise.

Gaussian noise. The results of this test are summarized, with the same notation used for Fig. 14, in Fig. 15, where the overall features retrace the features of the results obtained for a structured noise. However, some differences can be noticed. First, when unstructured noise is added to the reference heads, an overall degradation of the values of $\lambda^{2}$ can be observed (Fig. 15a). For the smallest gradients (cross markers) the curves do not differ much from the same curves corresponding to the structured noise, because even the structured noise was great enough to give unsatisfactory results for the identification of $T$, with $\lambda^{2} \simeq 3$. On the other hand, for the highest gradients (circle markers) the values of $\lambda^{2}$ deteriorate faster than for the structured noise. Concerning the MAE on $h$, the curves for the smallest gradients are comparable with the curves obtained for the structured noise, whereas the curves related to standard deviations greater than $5 \mathrm{~cm}$ (Fig. 15b) do not show a clear convergence pattern. 